\title{
ESTRATEGIAS EMPLEADAS POR LAS MADRES DURANTE EL PROCESO DE ALIMENTACIÓN EN NIÑOS DE 6 A 24 MESES DE DOS COMUNIDADES RURALES DE LIMA, PERÚ
}

\author{
Gandy Dolores-Maldonado1,a, Doris Delgado-Pérez²,b, Hilary Creed-Kanashiro ${ }^{3, c}$
}

\begin{abstract}
RESUMEN
Objetivos. Conocer las estrategias de la madre durante el proceso de alimentación en niños de 6 a 24 meses de dos comunidades de Lima. Materiales y métodos. Estudio cualitativo, participaron 18 madres primíparas o multíparas de niños de 6-24 meses de edad, se empleó un muestreo intencionado por edad y paridad de la madre, se realizaron 36 observaciones directas y 12 entrevistas a profundidad. Análisis de datos inductivo, las categorías se agruparon por temas y por técnica, llegando a un consenso de los temas entre autores. Resultados. La comunicación verbal se vio marcadamente más en las madres multíparas. Se encontraron tres grandes temas; comunicación verbal madre-hijo, las madres multíparas fueron quienes utilizaron palabras afectivas durante la comida. Involucramiento y estrategias para dar de comer se vio caracterizado por la animación al niño a terminar la comida mediante, juegos y cantos que mostraron las madres multíparas con ayuda, algunas veces, de otros integrantes de la familia; sin embargo, no se obtuvo los mismos resultados por parte de las madres primíparas, ellas generalmente se tornaron represivas ante el rechazo de la comida. El contexto durante la comida, favorece a que el niño termine su comida. Conclusiones. Las estrategias utilizadas durante el tiempo de comida fueron variadas y parcializadas; predominantemente para las madres multíparas, actuando de una forma más activa y responsiva, sin embargo, las madres primíparas pueden llegar a actuar de una forma autoritaria cuando el menor se ensucia, juega y/o rechaza los alimentos.
\end{abstract}

Palabras clave: Estrategia; Alimentación; Investigación cualitativa; Madres; Niño (fuente: DeCS BIREME).

\section{MOTHER'S STRATEGIES IN THE FEEDING PROCESS OF CHILDREN AGED 6 TO 24 MONTHS IN TWO RURAL COMMUNITIES OF LIMA, PERU}

\begin{abstract}
Objectives. To know the strategies of mothers during the feeding process in children aged 6 to 24 months in two communities of Lima. Materials and methods. Qualitative study in which 18 primiparous or multiparous mothers of children aged 6-24 months participated. We used intentional sampling by age and parity of the mother, and conducted 36 direct observations and 12 in-depth interviews. Inductive data analysis was conducted, categories were grouped by subject and technique, reaching a consensus of the themes among the authors. Results. Verbal communication was markedly greater in multiparous mothers. Three major themes were found. Verbal mother-child communication, multiparous mothers were those who used affectionate words during the meal. Involvement and strategies in the feeding process was seen as characterized by encouraging the child to finish the meal with games and singing that multiparous mothers performed sometimes with help from other family members. However these results were not obtained by primiparous mothers, they usually became withdrawn upon the rejection of food. The context during eating favors whether a child will finish the meal. Conclusions. The strategies used during meal time were varied and biased, predominantly by multiparous mothers, acting in a more active and responsive way. However primiparous mothers may act in an authoritarian manner when the child gets dirty, plays and/or refuses food.
\end{abstract}

Key words: Strategies; Feeding; Qualitative research; Mothers; Child (source: MeSH NLM)

Dirección de Salud II Lima Sur. Lima, Perú.

Universidad Nacional Mayor de San Marcos. Lima, Perú.

Instituto de Investigación Nutricional. Lima, Perú.

Nutricionista; ${ }^{\mathrm{b}}$ nutricionista, magíster en Nutrición; ${ }^{\mathrm{c}}$ nutricionista, magíster en Filosofía

La presente investigación forma parte de la tesis de licenciatura de Dolores Gandy "Alimentación perceptiva en niños desde los seis a veinticuatro meses de edad en el distrito de Cochamarca, Oyón - Lima 2014", presentada a la Facultad de Medicina de la Universidad Nacional Mayor de San Marcos, 2014.

Recibido: : 16-11-14 Aprobado: 19-08-15

Citar como: Dolores-Maldonado G, Delgado-Pérez D, Creed-Kanashiro H. Estrategias empleadas por las madres durante el proceso de alimentación en niños de 6 a 24 meses de dos comunidades rurales de Lima, Perú. Rev Peru Med Exp Salud Publica. 2015;32(3):440-8. 


\section{INTRODUCCIÓN}

El retardo del crecimiento o la desnutrición crónica sigue siendo un problema en varios países del mundo, especialmente en las zonas geográficas de mayor pobreza y menor desarrollo. En el Perú, la prevalencia de desnutrición crónica reportada en el 2014 en la zona rural $(21,9 \%)$, es más alta que la zona urbana $(5,8 \%)$ (1). Sin embargo, la magnitud del retardo del crecimiento no es uniforme, en los distritos más pobres las prevalencias son mayores ${ }^{(1)}$. Una de las causas es la inadecuada alimentación, las deficientes características sociodemográficas, bajo nivel educativo de la madre, pobreza, deficiencia de servicios básicos, entre otros ${ }^{(2)}$. Afectando los niveles de ingesta de energía y nutrientes para un buen crecimiento y desarrollo del niño ${ }^{(3)}$.

Pocos estudios han reportado el comportamiento del cuidador del niño durante el proceso de alimentación. Siendo este aspecto muy importante para un adecuado crecimiento y desarrollo del niño, principalmente los dos primeros años de vida, época en la cual el cerebro se forma y desarrolla a la máxima velocidad ${ }^{(4)}$. Si en estos períodos se altera, se producirán consecuencias que afectarán al niño durante toda su vida ${ }^{(5)}$. Creciendo inseguros, con baja autoestima y escasa motivación para progresar, incapacidad de dar afecto a otros, y con dificultades para manejar situaciones de conflicto ${ }^{(6)}$.

En la actualidad, y durante los últimos años, la OMS/ OPS ubica este tipo de alimentación, como responsiva, perceptiva o interactiva, una estrategia importante para una adecuada alimentación complementaria. Se recomienda que sea tanto sensible (poder leer las señales del niño), como responsiva (responder a las señales del niño), para un adecuado consumo de los alimentos ${ }^{(7)}$.

Las estrategias de estimulación se producen a través de interacciones apropiadas entre cuidadores y niños, que mejoran el consumo de alimentos y el desarrollo infantil (4,8). La madre debe lograr establecer una relación que influya en su consumo de alimentos, y minimizar el rechazo a la comida y la insistencia en dar de comer, evitando problemas de comportamiento alimentario ${ }^{(9)}$.

Esto hace que los primeros dos años de vida sea un tiempo ideal para ayudar a las madres a establecer hábitos saludables de alimentación y evitar la desnutrición así como el exceso de peso. Por ello, es importante tomar en cuenta también el cómo, dónde y cuándo alimentar al niño, así como las interacciones madre-niño ${ }^{(10)}$.

Existen, actualmente, escasos estudios en los pobladores de las zonas rurales del Perú, menos aun en nuestra propia capital que describan cómo es el proceso de alimentación y qué estrategias adoptan las madres para lograr que el niño tenga un suficiente y placentero consumo de alimentos. El objetivo del estudio fue conocer las estrategias de la madre durante el proceso de alimentación en dos comunidades rurales de Lima, Perú.

\section{MATERIALES Y MÉTODOS}

Estudio cualitativo con método de diseño narrativo (11), donde se buscó recoger datos a través de experiencias de las madres y observación del entorno durante el proceso de la alimentar a su niño. La población estuvo conformada por madres biológicas de niños entre 6 a 24 meses de edad, de dos comunidades del distrito de Cochamarca, ubicadas en una zona rural: San Juan de Yarucaya y Colcapampa, pertenecientes a la provincia de Oyón, del departamento de Lima, ambas ubicadas a 3620 metros de altitud; a $10 \mathrm{~h}$ de Lima para Cochamarca y a $9 \mathrm{~h}$ de Colcapampa por vía terrestre. Ambas comunidades tuvieron acceso limitado a servicios básicos, y fueron elegidas debido a que se presentó mayor accesibilidad a la zona, tanto climatológicamente como por parte de la comunidad.

La participación de la madre fue voluntaria, cada una firmó el consentimiento informado como aceptación de su participación. Los niños no presentaron ninguna enfermedad $u$ otro factor que interfiera con su alimentación o implique un cuidado mayor, por parte de la madre, durante el proceso de alimentación. El 100\% de las madres hablaban español.

El tipo de muestreo en cada comunidad fue primero intencionado y después por saturación técnica de la información. El muestreo intencionado agrupó a madres por paridad (primípara o multípara). La saturación técnica fue cuando ya no se encontraba la información adicional sobre los temas encontrados, obteniéndose una muestra final de 18 madres, nueve madres en cada comunidad.

Las madres participaron en las dos técnicas cualitativas, observación directa no participativa o entrevista a profundidad. La primera técnica se aplicó a doce madres, cada madre tuvo tres observaciones cada una, haciendo un total de 36 observaciones. Se determinó visitar su hogar por tres días no consecutivos, durante el tiempo que dure una de las comidas principales, sea en el desayuno, almuerzo o cena. El tiempo de duración de cada observación directa no participativa fue entre 20 a 45 minutos y de la entrevista a profundidad de 30 minutos, con un rango de 26 a 40 minutos. Al término de cada técnica se aplicó una encuesta de datos 
sociodemográficos, edad, paridad, nivel de escolaridad y acceso a servicios básicos en la vivienda. Las guías de preguntas fueron revisadas por jueces expertos de la Escuela Académico Profesional de Nutrición de la Universidad Nacional Mayor de San Marcos, habiendo una concordancia entre los jueces. También se aplicó una prueba piloto.

La guía de preguntas para la observación directa no participativa y la entrevista a profundidad, estuvo conformada por tres partes, 1). Comunicación verbal madre-hijo durante la comida; 2). Involucramiento y estrategias para animar a comer y 3). El contexto durante la comida.

Los datos fueron procesados con el software de análisis de datos cualitativos Atlas.ti (versión 7.1. 2011; Scientific Software Development), el análisis de datos empezó desde el primer día de la recolección de la información. Al finalizar cada día se transcribió a formato Word, las grabaciones de las entrevistas a profundidad (audios), notas escritas en la bitácora de capo y las observaciones directas no participativas (videos).

Tabla 1. Características generales de las madres, niños del distrito de Cochamarca $(n=18)$ y de la comida principal observada

\begin{tabular}{|c|c|c|}
\hline Características & $\frac{\text { Cochamarca }}{\mathrm{n}(\%)}$ & $\begin{array}{c}\text { Colcapampa } \\
\mathrm{n}(\%)\end{array}$ \\
\hline $\begin{array}{l}\text { Promedio de edad materna } \\
\text { (rango) }\end{array}$ & $26(20-32)$ & $27(21-35)$ \\
\hline Madres primíparas & 4 & 5 \\
\hline Madres multíparas & 5 & 4 \\
\hline \multicolumn{3}{|l|}{ Nivel educativo } \\
\hline Primaria incompleta & $2(11)$ & $1(6)$ \\
\hline Primaria completa & $2(11)$ & $3(17)$ \\
\hline Secundaria incompleta & $5(27)$ & $5(28)$ \\
\hline \multicolumn{3}{|l|}{$\begin{array}{l}\text { Servicios básicos en los } \\
\text { viviendas }\end{array}$} \\
\hline Agua de acequia, río & $8(44)$ & $8(44)$ \\
\hline Agua por red pública & $1(6)$ & $1(6)$ \\
\hline $\begin{array}{l}\text { Silo } \\
\text { Sin disposición de excretas }\end{array}$ & $\begin{array}{l}3(17) \\
6(33)\end{array}$ & $\begin{array}{l}4(22) \\
5(28)\end{array}$ \\
\hline \multicolumn{3}{|l|}{$\begin{array}{l}\text { Actividad principal a que se } \\
\text { dedica }\end{array}$} \\
\hline Su casa, ganadería y agricultura & $9(50)$ & $9(50)$ \\
\hline \multicolumn{3}{|l|}{ Niños } \\
\hline $\begin{array}{l}\text { Edad promedio en meses } \\
\text { (rango) }\end{array}$ & $16,0(8-22)$ & $15,0(7-21)$ \\
\hline \multicolumn{3}{|l|}{ Sexo } \\
\hline Masculino & $4(22)$ & $5(28)$ \\
\hline Femenino & $5(28)$ & $4(22)$ \\
\hline \multicolumn{3}{|l|}{ Comida principal observada } \\
\hline Desayuno & $0(0)$ & 0() \\
\hline Almuerzo & $12(33)$ & $11(31)$ \\
\hline Cena & $6(17)$ & $7(19)$ \\
\hline
\end{tabular}

Después de obtener todas las transcripciones, se empezaron a codificar para formar los temas, en un modelo adaptado de Glaser y Strauss teoría fundamentada ${ }^{(12)}$ y según Grahan Gibbs ${ }^{(13)}$. Se realizó la triangulación por técnica, para constatar que la información por ambas partes mantuviera el mismo mensaje. En el proceso de identificación de temas y códigos se tomó en cuenta las transcripciones de las emociones que se pudieron registrar durante ambas técnicas; también se analizaron las frases coloquiales de la zona para el mejor entendimiento. Los temas finales fueron interpretados y discutidos entre los investigadores, por consenso se llegó a los temas agrupados por características comunes y las citas más relevantes.

\section{RESULTADOS}

Una descripción de las características de las personas encargadas del cuidado del niño, quienes, en su totalidad, fueron las madres, se presenta en la Tabla 1. Las 18 madres tuvieron educación básica incompleta, ninguna de las viviendas contaba con servicios básicos de agua potable en red, ni conexión de desagüe. No se presentó mayores diferencias entre las dos comunidades, por el contrario, se fortalecieron durante el análisis de datos, de donde emergieron tres grandes temas.

\section{TEMA 1: COMUNICACIÓN VERBAL MADRE-HIJO DURANTE LA COMIDA}

En ambas comunidades, una de las estrategias que las madres multípara o primíparas utilizaron durante el proceso de la alimentación fue la comunicación verbal con su hijo, antes y durante el proceso de la alimentación.

Las madres multíparas, generalmente, se comunicaron de una forma interactiva (Tabla 2: 1.1 y 1.2), no sucedió lo mismo con la mayoría de las madres primíparas, ya que en lugar de ellas, actuaron otros integrantes de la familia como el abuelo, papá, primos o, hermanos (Tabla 2: 1.3 y 1.4$)$.

\section{TEMA 2: INVOLUCRAMIENTO Y ESTRATEGIAS PARA ANIMAR A COMER}

Las madres multíparas manifestaron utilizar diversas estrategias de involucramiento para animar a comer al niño durante el proceso de alimentación, actuando de una manera activa persuasiva. Una de las estrategias utilizada fue persuadir al niño para que coma, ofreciéndole comprar un producto que le gusta, como golosinas o bebidas azucaradas (Tabla 2: 2.1); otras madres manifestaron que motivaban a sus hijos a terminar su comida haciendo competencia con su hermano mayor (Tabla 2: 2.2). Ellos 
Tabla 2. Ejemplos de citas que apoyan al Tema 1: Comunicación verbal durante la comida y Tema 2: Involucramiento y estrategias para animar a comer

\begin{tabular}{|c|c|}
\hline Número & Tema / Citas \\
\hline 1 & Temas 1: Comunicación verbal durante la comida \\
\hline 1.1 & $\begin{array}{l}\text { l...Yo le tengo que cargar, porque ella se puede pasar jugando...., pero primero le digo hijita vamos a comer, ya hijita, } \\
\text { así le digo comida, hijita comida; vamos a comer, así no más yo le digo (EP, madre multípara con hija de } 10 \text { meses - } \\
\text { comunidad Cochamarca). }\end{array}$ \\
\hline 1.2 & $\begin{array}{l}\text { l...cuando ya es la hora de comer le hago sentar y le sirvo, hablando me hace caso cuando le digo agarra tu cuchara } \\
\text { mamita, pero siempre le ayudo un poco, cuando se distrae (EP, madre adulta multípara con hija de } 11 \text { meses - comu- } \\
\text { nidad Colcapampa). }\end{array}$ \\
\hline 1.3 & $\begin{array}{l}\text { Como no tengo mucho tiempo, no le estoy hablando mucho, pero con sus primos se juega, a veces come a veces no } \\
\text { (EP, madre joven primípara con hija de } 12 \text { meses - comunidad Cochamarca). }\end{array}$ \\
\hline 1.4 & $\begin{array}{l}\text { Yo a veces no le hablo mucho, más su abuelo que le hace juegos cuando come (EP, madre joven primípara con hijo } \\
\text { de } 11 \text { meses - comunidad Colcapampa). }\end{array}$ \\
\hline 2 & Tema 2: Involucramiento y estrategias para animar a comer \\
\hline 2.1 & $\begin{array}{l}\text { l... ya cuando no quiere total, nosotros le engañamos, diciendo que vamos comprar frugos, si terminas tu comida, así } \\
\text { a veces le decimos, de ahi se olvida pero.../ (EP, madre multípara con hija de } 23 \text { meses - Comunidad Cochamarca). }\end{array}$ \\
\hline 2.2 & $\begin{array}{l}\text { I... yo siempre como con mis dos hijos y al más chiquito le digo, hijito mira tu hermano -tiene } 9 \text { años - te va ganar termina } \\
\text { tu comida, entonces por querer ganarle ya come solo su comida y el que termina primero, voltea su plato y le digo muy } \\
\text { bien campeón (EP madre multípara con hijo de } 22 \text { meses - Comunidad Cochamarca). }\end{array}$ \\
\hline 2.3 & $\begin{array}{l}\text { l...cuando a veces no quiere comer, con sus juguetes le hago distraer, sus hermanos me ayudan, como van al colegio } \\
\text { aprenden a cantar y cuando ellos cantan, mi hijito se distrae, ahí le hago comer un poco más (EP, madre multípara con } \\
\text { hijo de } 20 \text { meses - comunidad Colcapampa). }\end{array}$ \\
\hline 2.4 & $\begin{array}{l}\text { I...cuando no quiere, come mitad no más, allí le deja o saca su comida con su mano y después juega, por eso yo le car- } \\
\text { go y le hago comer de nuevo, yo le hago terminar a la mala, le hecho la comida no más, hace maña para que coma.../ } \\
\text { (EP, madre primípara con hijo de } 18 \text { meses - Comunidad Cochamarca). }\end{array}$ \\
\hline 2.5 & $\begin{array}{l}\text { l...como ya no me da mucho tiempo, le dejo, pero cuando come muy poquito, ahí si le tengo que dar rápido en su } \\
\text { boca, sino se mal acostumbra y puede ser un malcriado, ya después sigo haciendo las cosas de la casa.../(EP, madre } \\
\text { primípara con hijo de } 12 \text { meses - Comunidad Colcapampa), }\end{array}$ \\
\hline
\end{tabular}

también ayudaron a motivar a su hermano menor a través del canto (Tabla 2: 2.3). Mediante la observación directa no participativa se registró aspectos similares en ambas comunidades, utilizando estrategias para animar a comer, tales como: el canto y el juego con los utensilios o con la comida, comportándose de una forma interactiva, algunas veces con ayuda de otros miembros de la familia; por otro lado, las madres priorizaron el servido de la comida a la familia y algunas obligaciones de casa, antes de continuar atendiendo a sus hijos pequeños, pero se preocupaban que sean bien atendidos.

Las madres primíparas en ambas comunidades manifestaron que no juegan con el menor durante el proceso de alimentación, hubo un escaso uso de palabras amables y afectivas (Tabla 2: 2.4 y 2.5). Se observó que ante la acción del rechazo de la comida o cuando el niño tomaba mucho tiempo para comer, ellas mostraron respuestas represivas y/o amenazantes.

\section{TEMA 3: EL CONTEXTO DURANTE LA COMIDA}

Subtema 3.1. Ambiente del proceso de alimentación:

Las madres multíparas manifestaron utilizar estrategias de cambiar el ambiente para el proceso de alimentación del niño, tales como llevar las preparaciones para que el niño coma fuera de casa, en el campo (Tabla 3: 3.1.1). Se caracterizó como importante por la mayoría de las madres multíparas; ya que era más probable que el niño termine la comida e, incluso, a veces comió un poco más. Manifestaron que no existió rechazo a la comida, debido a que se entretiene mirando plantas y/o animales, además que tienen el apoyo de sus otros hijos para alimentarlo, pero esta estrategia le demandó mayor tiempo.

En las dos comunidades las madres primíparas jóvenes manifestaron todo lo contrario, no salir fuera de casa para que coma su hijo. Refirieron que sus hijos casi nunca comen en el campo, a pesar de que ellas salen de casa, regresan a preparar la comida, considerándolo tedioso, comer fuera de casa (Tabla 3: 3.1.2). En cambio, las madres primíparas adultas manifestaron que algunas veces sí llevaban a sus hijos a comer fuera de casa. Refirieron que dejan que el termine su comida, no involucrándose en el proceso de la alimentación (Tabla 3: 3.1.3).

Subtema 3.2. Exploración de la comida con sus manos:

Mediante las dos técnicas aplicadas se registró que, generalmente, las madres primíparas fueron quienes no permitieron a sus hijos explorar los alimentos con sus 
Tabla 3. Ejemplos de citas que apoyan al Tema 3: El contexto durante la comida

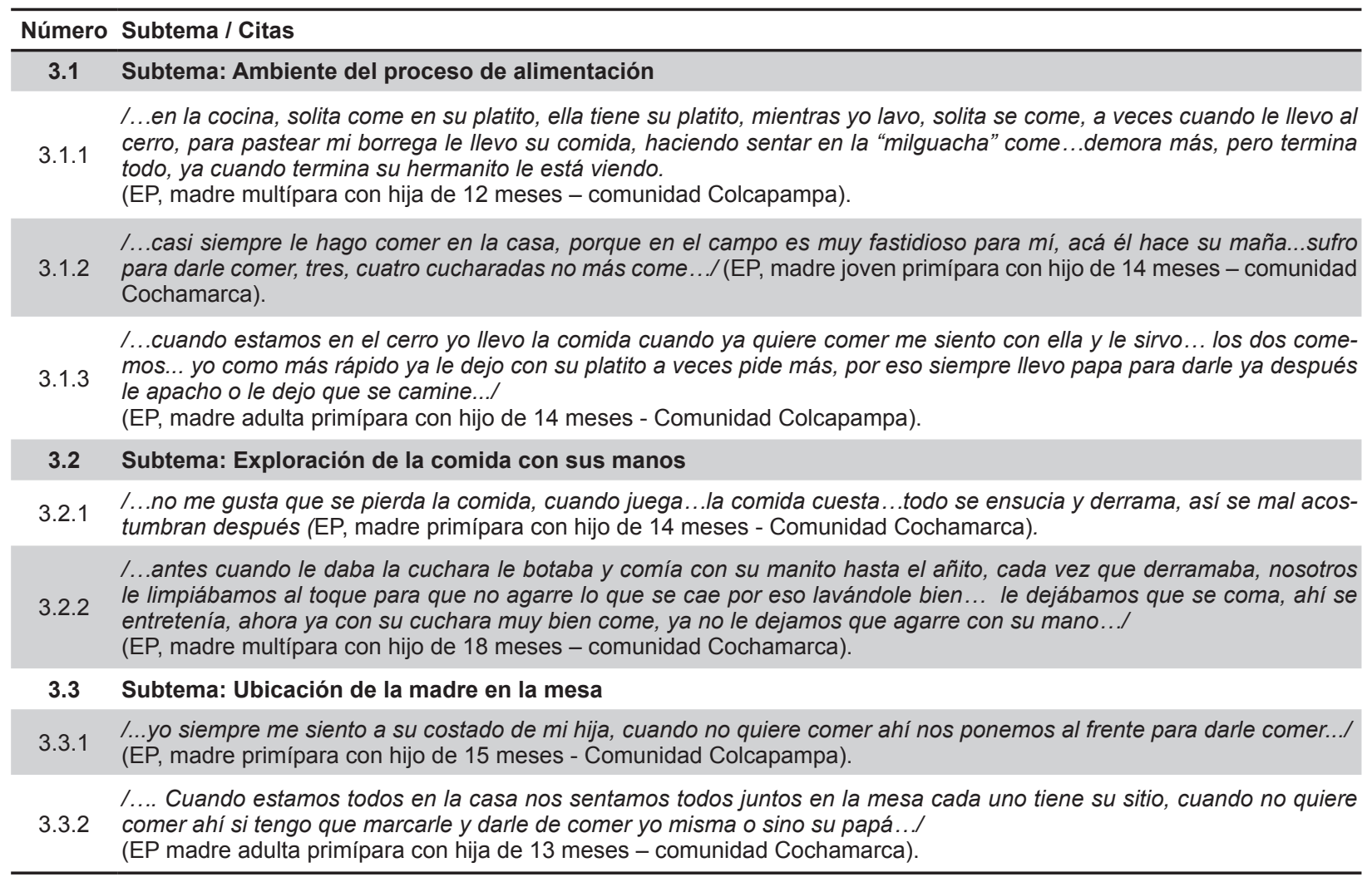

manos. Se caracterizó por tener una actitud autoritaria, a fin de evitar que su hijo ensucie la ropa, la mesa y el desperdicio de alimentos que las madres consideran costosos, como el arroz (Tabla 3: 3.2.1).

Las madres multíparas presentaron una actitud responsiva o interactiva con su hijo, ante el intento de la exploración de alimentos con sus manos, permitiéndole tocar, jugar y combinarlos (Tabla 3: 3.2.2).

\section{Subtema 3.3. Ubicación de la madre en la mesa}

La ubicación de las madres en la mesa durante el proceso de la alimentación, fue al costado de sus hijos (Tabla 3: 3.3.1) y solo estaban frente a él cuando se hacía necesario asistirlo (Tabla 3: 3.3.2). Mediante la técnica de observación se encontró que algunas madres asistían a sus hijos sin estar frente a frente y sin un contacto visual directo.

\section{DISCUSIÓN}

Los resultados mostraron tres temas emergentes, la comunicación verbal durante la comida, el involucramiento y estrategias para animar a comer y el tercer tema: contexto durante la comida, con tres subtemas: ambiente del proceso de alimentación, exploración de la comida con sus manos y ubicación de la madre en la mesa.

La comunicación entre madre e hijo se produjo, principalmente, de una manera verbal y en tiempos diferentes, antes y durante el proceso de alimentación. Los estímulos verbales se reconocen en mayor medida como medios facilitadores del proceso de crianza con miras a la formación integral de los niños y en especial el desarrollo del lenguaje ${ }^{(14)}$. Esto puede verse afectado si es que entre el niño y la madre no existe una fuerte conexión comunicacional; esto sucedió predominantemente en las madres primíparas jóvenes, en quienes se evidenció que la comunicación durante la comida fue corta y poco afectiva, es decir, menos involucrada, ya que en lugar de ellas, otros integrantes de la familia propiciaban hablar con el menor. Affleck y Pelto en Bangladesh encontraron que algunas de las madres no vieron como un facilitador el comunicarse con sus hijos, sino como una pérdida de tiempo, ya que preferían invertir aquel tiempo en realizar otras actividades del hogar que priorizaban como más importantes $^{(15)}$.

Un estudio con enfoque mixto realizado en Vietnam por Caulfield et al. mencionan que menos de la mitad de 
los cuidadores que hablaron con sus hijos durante la comida fueron sensibles en el tono de las palabras (16). Moore et al. presentaron en su estudio observacional que la comunicación durante el tiempo de comida entre cuidador y niños era escasa y las pocas interacciones fueron sobre temas que no tenían relación con los alimentos (17). Estos patrones son similares a lo observado en este estudio, que también mostró escasa comunicación por parte de la madre debido principalmente a la falta de tiempo, ya que destinan en mayor cantidad al trabajo doméstico y la agricultura, restando valor a la cantidad y calidad del tiempo para alimentar a los niños ${ }^{(18,19)}$.

Por el contrario, las madres multíparas, principalmente, mostraron una actitud más interactiva y afectiva, promoviendo la alimentación del menor. Sally Wiggins en su estudio menciona que aquellos padres que suelen utilizar palabras como "buena" o "deliciosa" en torno a la comida, podrían evitar que los niños respondan negativamente a la comida más adelante ${ }^{(20)}$.

Las madres multíparas priorizaron primero el servido de la comida a la familia y algunas obligaciones antes de atender a sus hijos pequeños, sin embargo, se preocuparon porque sean bien atendidos pidiendo ayuda al esposo o a sus hijos mayores y después de terminar de servir continuaron atendiendo al menor. Ellas fueron quienes en primera instancia fueron las más involucradas en la alimentación.

Generalmente el propósito de las madres durante la alimentación fue lograr que sus hijos terminen su comida sin demora, sin embargo, para lograr este propósito se actuó de diferentes formas; desde una forma responsiva, interactiva, acompañada de juegos, motivaciones hasta una forma autoritaria y forzada.

Las madres multíparas fueron las que mostraron en mayor grado estrategias de alimentación, ayudando a propiciar una alimentación suficiente y amena a base de motivaciones, juegos, gestos y demostraciones, haciendo uso de estrategias como decir palabras afectivas, alentadoras y un tanto competidoras. Wondafras et al. refieren que los niños comen más cuando se alienta verbalmente ${ }^{(21)}$, igualmente, en Cochamarca el involucramiento interactivo con el menor, resulta beneficioso para un consumo mayor consumo.

Asimismo, el apoyo para alimentar también estuvo presente cuando el niño era más pequeño, donde actúan otros integrantes de la familia (22). Según Aubel ${ }^{(23)}$ el apoyo de otros miembros de la familia y de la comunidad, tales como las abuelas, hermanas, tías, etc. también debe ser aprovechada para crear un entorno propicio para que las madres alimenten y cuiden a sus hijos(as) debidamente. Vazir et al. mostraron que mediante una intervención a madres por medio de mensajes de alimentación complementaria y de alimentación responsiva del uso de juguetes para la estimulación, mejoraron los niveles de ingesta alimentaria ${ }^{24)}$. En el Perú se realizó también una intervención parecida, mejorando la nutrición de los niños ${ }^{(25)}$. Asimismo, en Ghana se observó por seis meses cómo la capacidad de respuesta del cuidador durante la alimentación fue significativamente mayor para el consumo de alimentos, con una mejoría del estado nutricional, a diferencia de aquellos niños cuyas madres no eran muy sensibles a las necesidades de sus hijos ${ }^{(26)}$. Haycraf et al. aseveran que el modelado de los padres en los comportamientos y actitudes en la alimentación está relacionado con la capacidad de respuesta de los niños, como el interés, deseo, disfrute o rechazo de los alimentos ${ }^{(27)}$.

Por ello, se debe propiciar el uso de estrategias que permitan la mejor aceptación de los alimentos que serán, en primera instancia, función de la persona a cargo del cuidado del niño y serán relevantes para convertir un hábito en algo recurrente ${ }^{(28)}$.

Por otro, lado las madres primíparas mostraron escasas palabras amables y afectivas, sin juegos y/o demostraciones, en cuanto al rechazo de la comida por parte del menor, ellas llegaron a ser represivas y algunas veces amenazantes. En Cochamarca se encontraron casos en los que se forzó al niño a comer, cuando rechazaba la comida volteando la cabeza; la mayoría de las madres primíparas optaron por esta actitud argumentando que si permitían que realicen berrinches, sus hijos se malacostumbrarían y serían más adelante malcriados. Moore et al. evidenciaron que solo la tercera parte es sensible a la práctica de alimentación activa ${ }^{(29)}$, es decir, anima al niño a comer a través de palabras, mientras otras cuidadoras alimentaron a sus hijos directamente de una manera forzada. Un estudio realizado en Bangladesh se caracterizó por inducir presión para lograr alimentar al menor, lo que , a la vez, se asoció con el rechazo hacia los alimentos (21,30). Forestell et al. mencionan que a mayor paciencia por parte de la madre en ofrecer repetidamente los alimentos, disminuye el rechazo, pero con una mayor demanda de tiempo ${ }^{(31)}$.

Por otro lado, el dar de alimentar forzadamente disminuía el tiempo durante la comida, dejando mayor tiempo para otras actividades. Bentley, mediante la observación directa en el hogar, mostró que la alimentación forzada tomó mucho menos tiempo que una alimentación asistida con interacciones entre madre-niño ${ }^{(16)}$, al igual que en Cochamarca, para las madres primíparas la inversión de tiempo en las actividades de la casa poseen mayor prioridad que el tiempo para alimentar a sus hijos. 
Dentro del contexto durante la comida, la mayoría de las madres multíparas manifestaron que es importante el lugar dónde come, porque existen ventajas cuando el menor se distrae en el campo, facilitando el consumo total de sus alimentos, aunque les lleve mayor tiempo alimentarlo. Asimismo, permitieron la exploración de los alimentos con sus manos, actuando de una forma responsiva, esta actitud estuvo caracterizado por las madres multíparas; similarmente, Hetherington et al. evidenciaron que mediante grupos focales y entrevistas a profundidad las madres manifestaron al destete como una oportunidad fundamental para el desarrollo de los niños, fundamentado en la introducción de nuevos sabores, incluyendo verduras. La exposición del sabor y el descubrimiento al gusto durante el destete fueron identificados como el comienzo de un "un recorrido del sabor", en la que educar al paladar con una variedad de diferentes alimentos se consideró importante ${ }^{(32)}$.

Las madres primíparas refirieron que sus hijos permanezcan en casa, ya que fue más fácil alimentarlos en una mesa y tenerlos más controlados y así evitaban que se ensucie, o juegue con los alimentos, imposibilitando la exploración de alimentos con sus manos. Asimismo, mostraron una actitud autoritaria ante el intento de la exploración de los alimentos por parte del niño. Gómez et al. también encuentran la escasa presencia del diálogo o explicación frente al castigo o represalia que se impone, evidenciándose una regulación negativa basada en métodos autoritarios ${ }^{(33,34)}$. Un estudio reciente realizado en Malawi encuentra que el control de la conducta durante la alimentación se asocia negativamente con la aceptación de los alimentos por el niño ${ }^{(17)}$.

En general, los tres temas se han visto afectados en algún momento por el factor tiempo, el cual impidió que las madres, principalmente primíparas, actuaran de una forma responsiva, por ende, escasas prácticas de estrategias para alimentar responsivamente, priorizando la actividad agrícola - ganadera, las cuales fueron la fuente principal de ingreso, en Bolivia también se evidencian estas prácticas ${ }^{(15,22)}$.

El presente estudio presenta algunas limitaciones que deben de considerarse para futuras investigaciones en el tema. El tamaño de muestra fue limitado y podría extenderse para una mayor riqueza de datos; no se consideró el estado emocional de la madre, pero puede haber influido en el comportamiento, ya que por su carga laboral muchas veces se veían presionadas y posiblemente exista un nivel de estrés, además, la ausencia o presencia del apoyo familiar.

En el estudio se concluye que existe una variedad de estrategias que se realizaron durante el proceso de la alimentación. Las madres multíparas fueron quienes se caracterizaron por mostrar mayoritariamente estrategias responsivas, promoviendo una comunicación verbal afectiva, la motivación mediante juegos y cantos, llevar al niño a comer fuera de casa y permitir la exploración de alimentos, algunas veces apoyadas por diferentes miembros de la familia. Por el contrario las madres primíparas, generalmente se mostraron pasivas para la comunicación y motivación a comer y autoritarias para evitar la exploración de alimentos. Vale mencionar que ninguna de las madres no tuvo contacto directo ojo a ojo mientras se hablaba o se asistía al niño, faltando a los principios de cuidado psicosocial ${ }^{(35)}$.

Las madres multíparas se muestran como las personas clave para próximas intervenciones efectivas educacionales en infantes, debido a que poseen un mayor vínculo afectivo, en el uso de estrategias, así también los otros integrantes de la familia, quienes muestran apoyo. Esta investigación es precedente para que más adelante se pueda realizar intervenciones educativas responsivas longitudinales o investigaciones con diseños etnográficos, donde se pueda tener una visión mucho más amplia del entorno familiar teniendo en cuenta también variabilidad sociocultural, estado emocional de la madre y seguridad alimentaria de cada conjunto de personas, para identificar mejores formas de integrar la alimentación complementaria con la alimentación responsiva y lograr un cambio en el comportamiento.

Contribuciones de autoria: GDM ha participado en la concepción y diseño del artículo, la recolección de datos, análisis e interpretación de datos, redacción final y obtención del financiamiento parcial. Además DDP realizó la asesoría durante todo el proceso de la investigación y HCK en la revisión crítica del artículo.

Fuentes de financiamiento: este estudio fue parcialmente financiada por el "Fondo de tesis de pregrado 2013" según RD 0214-D-FM-2013 de la Universidad Nacional Mayor de San Marcos.

Conflicto de interés: los autores no tienen conflictos de interés en la publicación de este artículo.

\section{REFERENCIAS BIBLIOGRÁFICAS}

1. Instituto Nacional de Estadística e Informática. Indicadores de resultados de los programas estratégicos, 20072014. Encuesta demográfica y de salud familiar (resultados preliminares). Lima: INEI; 2015.
2. Apaza-Romero D, Celestino-Roque S, Tantaleán-Susano K, Herrera-Tello $M$, Alarcón-Matutti E, Gutiérrez C. Sobrepeso, obesidad y la coexistencia de desnutrición crónica en niños menores de 5 años. Rev Peru Epidemiol. 2014;18(2):1-5.
3. Miranda M, Rojas J, Riega V, Valenzuela R, Cavero S, Maldonado R. Tendencias en el consumo de energía y nutrientes de niños peruanos menores de 5 años en el periodo 1997-2001. Rev Peru Med Exp Salud Publica. 2004;21(4):240-52. 
4. Grantham-McGregor S, Cheung YB, Cueto S, Glewwe P, Richter, Strupp B, et al. Developmental potential in the first 5 years for children in developing countries. Lancet. 2007;369(9555):60-70.

5. Feinstein L, Sabates R, Sorhaindo A, Rogers I, Herrick D, Northstone K, et al. Dietary patterns related to attainment in school: the importance of early eating patterns. J Epidemiol Community Health. 2008;62(8):734-9.

6. Kim JM, Mahoney G. The effects of relationship focused intervention on Korean parents and their young children with disabilities. Res Dev Disabil. 2005;26(2):117-30.

7. Organización Panamericana de la Salud. Principios de orientación para la alimentación complementaria del niño amamantado. Washington DC: OPS; 2003.

8. Arjumand S, Lori G, Hertzman C. Total environment assessment model of early child development. Vancouver: Human Early Learning Partnership; 2007.

9. Keren M, Feldman R, Tyano S. Diagnoses and interactive patterns of infants referred to a communitybased infant mental health clinic. J Am Acad Child Adolesc Psychiatry. 2001;40(1):27-35.

10. Black M, Creed-Kanashiro H. ¿Cómo alimentar a los niños? La práctica de conductas alimentarias saludables desde la infancia. Rev Peru Med Exp Salud Pública. 2012;29(3):373-8.

11. El proceso de la investigación cualitativa. En: Hernandez R, Fernandez C, Baptista P. Metodología de la investigación. 4ta ed. Mexico D.F.: McGraw-Hill Interamericana; 2006. p. 521-748.

12. Glaser B, Strauss A. The discovery of grounded theory: Strategies for qualitative research. 8va ed. New York: Aldine Transaction; 1967.

13. Gibbs G. Analyzing Qualitative Data. London: SAGE Publication; 2007.

14. Craig G. Desarrollo Psicológico. 6ta ed. México: Prentice Hall; 1992.

15. Affleck W, Pelto G. Caregivers' responses to an intervention to improve young child feeding behaviors in rural Bangladesh: A mixed method study of the facilitators and barriers to change. Soc Sci Med. 2012;75(4):651-8. doi: 10.1016/j.socscimed.2012.03.030.
16. Ha PB, Bentley ME, Pachón $H$, Sripaipan T, Caulfield LE, Marsh $\mathrm{DR}$, et al. Caregiver styles of feeding and child acceptance of food in rural Viet Nam. Food Nutr Bull. 2002;23(4 Suppl):95-100.

17. Moore AC, Akhter S, Aboud FE. Responsive complementary feeding in rural Bangladesh. Soc Sci Med. 2006;62(8):1917-30.

18. Blackden CM, Wodon Q. Gender, time use, and poverty in Sub-Saharan Africa. Report No.: 73. Washington: The International Bank for Reconstruction and Development; 2006.

19. Levinson M, Halpern O, Mahmud Z, Chowdhury S, Levinson J. Tufts Nutrition. Nutrition-related caring practices and women's time constraints: A study in rural Bangladesh [Internet]. Boston: 2002 [citado el 15 de Julio de 2015]. Disponible en: http://www.nutrition. tufts.edu/documents/fpan/wp18women_constraints.pdf

20. Wiggins S. Adult and child use of love, like, don't like and hate during family mealtimes. Subjective category assessments as food preference talk. Appetite. 2014;80:7-15. doi: 10.1016/j.appet.2014.04.024.

21. Wondafrash M, Amsalu T, Woldie M. Feeding styles of caregivers of children 6-23 months of age in Derashe special district, Southern Ethiopia. BMC Public Health. 2012 12:235. doi: 10.1186/1471-2458-12-235.

22. Jones A, Cruz Y, Galway L, Bentley J, Pinstrup-Andersen P. Heavy agricultural workloads and low crop diversity are strong barriers to improving child feeding practices in the Bolivian Andes. Soc Sci Med. 2012 Nov;75(9):1673-84.

23. Judi A. The roles and influence of grandmothers and men [Internet]. Washington D.C.: USAID; 2011 [citado el 15 de Julio de 2015]. Disponible en: http://www.iycn. org/files/IYCN-GM-and-Men-LitReview-060311.pdf

24. Vazir S, Engle P, Balakrishna N, Griffiths PL, Johnson SL, CreedKanashiro $\mathrm{H}$, et al. Cluster randomized trial on complementary and responsive feeding education to caregivers found improved dietary intake, growth, and development among rural Indian toddlers. Matern
Child Nutr. 2013;9(1):99-117. doi: 10.1111/j.1740-8709.2012.00413.x.

25. Penny ME, Creed-Kanashiro HM, Robert RC, Narro MR, Caulfield LE, Black RE. Effectiveness of an educational intervention delivered through the health services to improve nutrition in young children: a clusterrandomised controlled trial. Lancet. 2005;365(9474):1863-72.

26. Nti C, Lartey A. Effect of caregiver feeding behaviours on child nutritional status in rural Ghana. International Journal of Consumer Studies. 2007;31(3):303-9.

27. Palfreyman Z, Haycraf E, Meyer C. Parental modelling of eating behaviours: Observational validation of the Parental Modelling of Eating Behaviours scale (PARM). Appetite. 2015;86:31-7. doi: 10.1016/j. appet.2014.08.008.

28. Guzmán R, Gómez G, García R, Martínez JP. Una cuestión de género: el estado nutricional de los hijos e hijas, explicado desde las prácticas de crianza de las madres de familia. Revista científica electrónica de psicología. 2007.

29. Dettwyler K. Styles of Infant Feeding: Parental/Caretaker Control of Food Consumption in Young Children. Am Anthropol. 1989 October;91(3):696-703.

30. Bentley ME, Wasser HM, CreedKanashiro HM. Responsive feeding and child undernutrition in low- and middle-income countries. J Nutr. 2011;141(3):502-7. doi: 10.3945/ jn.110.130005.

31. Forestell CA, Mennella JA. More than just a pretty face. The relationship between infant's temperament, food acceptance, and mothers' perceptions of their enjoyment of food. Appetite. 2012;58(3):1136-42. doi: 10.1016/j. appet.2012.03.005.

32. Schwartz C, Madrellea J, Vereijken CM, Weenen $\mathrm{H}$, Nicklausd S, Hetherington MM. Complementary feeding and "donner les bases du goût" (providing the foundation of taste). A qualitative approach to understand weaning practices, attitudes and experiences by French mothers. Appetite. 2013;71:321-31. doi: 10.1016/j. appet.2013.08.022.

33. Vera J, Peña M. Desarrollo, estimulación y estrés de la crianza en infantes rurales 
de México. Apuntes de Psicología. 2005;23(3):305-19.

34. Gómez LF. Conducta alimentaria, hábitos alimentarios y puericultura de la alimentación. CCAP [Internet]. 2008 [citado en Mayo del 2012]; 7(4):38-50. Disponible en: http:// scp.com.co/descargascrianza/ Conducta\%20alimentaria, \%20
h\%C3\%A1bitos\%20alimentarios\%20 y\%20puericultura\%20de\%20la\%20 alimentaci\%C3\%B3n.pdf

35. Creed-Kanashiro H, Bartolini R. Proyecto de investigación "Alimentación responsiva en el Perú: aplicación y monitoreo de una intervención en familias con niños pequeños de una zona de la región
Ucayali". Informe final. Ucayaly: Instituto de Investigación Nutricional; 2009.

Correspondencia: Gandy Dolores Maldonado Dirección: Av. Puente Piedra 551 Lima.

Teléfono: (+511) 951590668

Correo electrónico:dgandykerlin@gmail.com

\section{http://twitter.com/rpmesp}

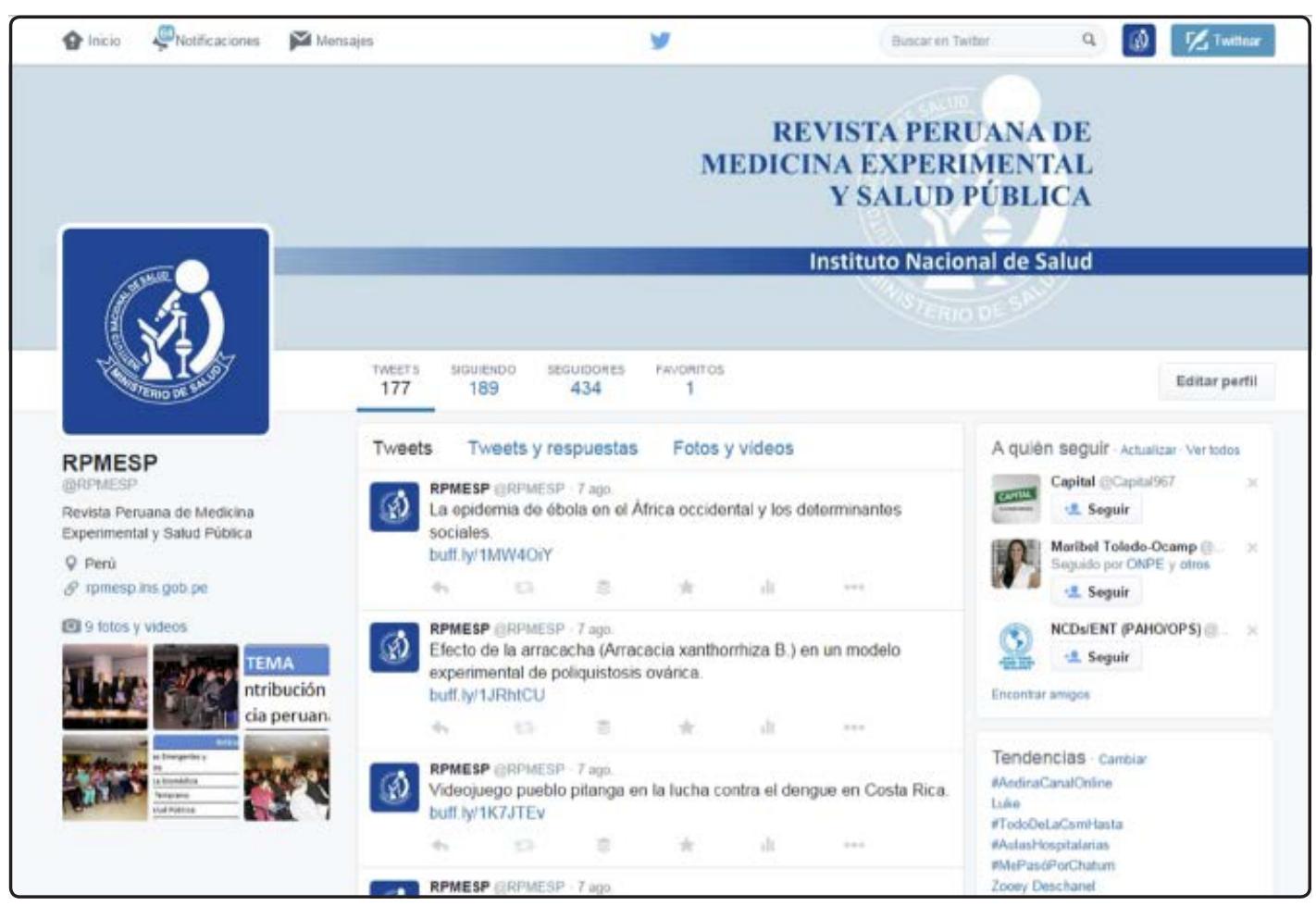

